\title{
Creando vínculos entre Universidad y Comunidad: el proyecto Shere Rom, una experiencia de aprendizaje servicio en la Facultad de Psicología de la Universitat Autònoma de Barcelona
}

\author{
José Luis Lalueza, Sònia Sánchez-Busqués y Marta Padrós \\ Universitat Autònoma de Barcelona
}

\section{Resumen}

En este trabajo se describe el proyecto Shere Rom, un entramado de vínculos entre la Universitat Autònoma de Barcelona, comunidades gitanas y escuelas públicas del área metropolitana de Barcelona. En el análisis de la experiencia se destacan dos elementos clave del aprendizaje servicio: a) el proyecto promueve el retorno social de la universidad a la sociedad, dando respuesta a necesidades reales mediante la intervención psicoeducativa desarrollada en el marco de proyectos de investigación; b) promueve en los estudiantes la adquisición de conocimientos y el desarrollo de competencias propias de su currículum formativo a través de la participación en un proyecto real y la implicación en la comunidad. Después de 16 años, participando cada curso entre 110 y 140 estudiantes de la UAB y entre 250 y 300 niñas y niños de distintas minorías étnicas, podemos describir las potencialidades que el proyecto ofrece a cada uno de sus participantes.

\section{Palabras clave}

Aprendizaje servicio, grupos minoritarios, gitanos, vínculo universidad-comunidad.

Fecha de recepción: 17/VIII/2014

Fecha de aceptación: 30/VII/2015 


\title{
Creating links between University and Community: Shere Rom's project, a service learning experience at the School of Psychology at the Universitat Autònoma Barcelona
}

\begin{abstract}
In this article we describe the project Shere Rom, a network of links between the Autonomous University of Barcelona, gypsie communities and public schools in the metropolitan area of Barcelona. The analysis of the experience highlights two key elements of service learning: a) the project promotes the social return of the university to society, responding to real needs through educational intervention that is developed under the frame of research projects; b) promotes, in college students, the acquisition of knowledge and the development of its own training curriculum skills through participation in a real project and community involvement. After 16 years, with a participation each year between 110 and 140 UAB students and between 250 and 300 children from different ethnic minorities, we can describe the potential that the project offers to each of its participants.
\end{abstract}

\section{Keywords}

Service Learning, minority groups, gypsies, univerisity - communitiy links. 


\section{Necesidades sociales que justifican el proyecto}

En algunos países, como los escandinavos, existe un amplio consenso en que la universidad tiene tres misiones. Las dos primeras son obvias: investigación y docencia, pero hay además una tercera misión definida como retorno a la sociedad. Se trata de una área similar a la que en España Ilamamos transferencia de conocimiento, con el imperativo ético de retornar a la sociedad la inversión que ésta hace a la universidad (gratuita o casi gratuita para sus estudiantes). La universidad así, debe promover acciones que supongan beneficios para la sociedad que vayan más allá de la construcción de nuevo conocimiento y de la formación de sus estudiantes.

El aprendizaje servicio (ApS) es un tipo de actividad generada por las universidades que tiene el potencial de abarcar las tres misiones, cubriendo necesariamente al menos dos de ellas, la docencia y el retorno social. Eso la distingue de otros tipos de actividad que pueden hacer los estudiantes bajo el paraguas de su universidad. Así, no es lo mismo que voluntariado, ya que éste puede tener elementos de retorno social, pero no responde a una actividad docente ni está diseñada o regularizada en torno a la adquisición de conocimientos y competencias que formen parte del currículo de la titulación correspondiente. También se diferencia de las prácticas externas, que sí responden a una forma de docencia, pero no incluyen como finalidad el componente de retorno social. 


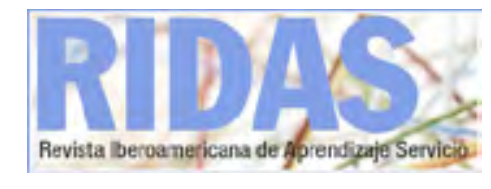

El proyecto Shere Rom es un proyecto de ApS de la Facultad de Psicología de Ia Universidad Autónoma de Barcelona, transversal a los ámbitos de investigación, docencia y retorno social, que pretende dar respuesta a tres tipos de necesidades sociales relacionadas con los procesos educativos: a) la necesidad de revisar el modelo escolar tradicional, b) la necesidad de transformar las prácticas educativas para favorecer la inclusión de miembros de grupos culturales minoritarios, y c) la necesidad de una nueva forma de afrontar la formación de los estudiantes universitarios para acercarla a prácticas en contextos reales.

Este proyecto utiliza el ApS como una herramienta no sólo para responder a cada una de estas necesidades, sino también para aunar en una misma actividad las tres misiones de la universidad: la investigación (de nuevas formas de organizar el a prendizaje), la docencia (aproximando a los estudiantes universitarios a procesos reales en entornos auténticos) y la transferencia de conocimiento a la sociedad (generando proyectos de intervención con capacidad para operar transformaciones sociales).

\subsection{Necesidad de revisar el modelo escolar tradicional}

Todas las sociedades organizan de algún modo la transferencia de experiencias y conocimientos de una generación a la siguiente. En las sociedades occidentales esta tarea se ha desarrollado históricamente en el marco de una institución, la escuela, basada en el modelo taylorista que se 


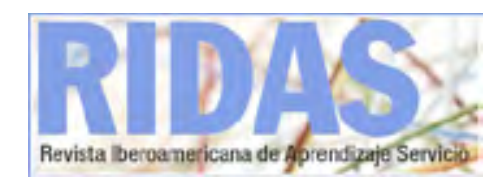

caracteriza por la producción estandarizada. Los estudiantes son procesados mediante un material estandarizado (currículum y libros de texto) con la meta de que adquieran los conceptos y competencias considerados básicos, y las formas de lenguaje y comportamiento deseables en quienes deben emplear su vida de adultos en vivir y trabajar en la sociedad industrial (Gatto, 2000).

Todavía hoy, en gran parte de las aulas escolares el aprendizaje se limita a un proceso de trasmisión o reproducción cultural, es decir, a recibir, recordar y reproducir lo que el maestro enseña. Las herramientas que se utilizan para el aprendizaje como trasmisión son principalmente los libros de texto y el discurso del profesor (Rogoff, 1994). Este tipo de escuela se caracteriza por un sólo maestro en el aula, en la que trabaja con un conjunto limitado de herramientas a su disposición para transmitir una limitada cantidad de información.

Este modelo de escuela tradicional de transmisión y almacenamiento de información puede que nunca haya descrito adecuadamente el proceso de enseñanza y aprendizaje (Säljö, 2000), pero es evidente que hoy está en crisis. Enunciados de la sociedad de la información y el conocimiento tales como aprendizaje a lo largo de toda la vida, flexibilidad, colaboración, creatividad y globalización, parecen no encajar con el modelo tradicional de enseñanza orientado a transmitir a los alumnos un único canon homogéneo. La enseñanza en un mundo en rápida evolución en el que proliferan 
distintas perspectivas debería ser muy diferente del proyecto de enseñar destrezas básicas para los alumnos de la comunidad local, o la enseñanza de los comportamientos requeridos para el trabajo industrial. Las tecnologías de la información y la comunicación (TIC), recientemente incorporadas a la escuela, tienen el potencial de ser herramientas educativas que atiendan a la diversidad en contenidos y vías de acceso al conocimiento, y permiten la introducción de múltiples perspectivas respecto a los objetos de conocimiento. Pero, a pesar de la llegada de las TIC a la escuela, los viejos modelos de enseñanza y aprendizaje parecen resistirse y el ordenador tiende a a ser utilizado como las viejas herramientas, como un instrumento de trasmisión más que como herramienta de indagación y creación (Crook, 1991).

Pero una nueva escuela no sólo precisa de un uso apropiado de las TIC, debe buscar nuevas formas de asociación con diferentes sectores de la sociedad que amplíen los fondos de conocimiento (Moll et al., 1991; Reyes y Esteban-Guitart, 2013) a los que puede acceder. Las organizaciones se transforman mediante procesos de aprendizaje expansivo, concepto generado por el enfoque histórico-cultural de la psicología y desarrollado por Engeström (1999), que entiende el aprendizaje como el desarrollo de nuevos conceptos, herramientas y actividades en respuesta a las cambiantes necesidades y contextos. Los cambios en los contextos generan contradicciones con los modos de operar previos a esos cambios, y la 
toma de conciencia de esas contradicciones, que se expresa a través de conflictos, puede generar procesos de aprendizaje expansivo, y por lo tanto de cambio en la organización. El aprendizaje así entendido no puede ser fruto de una única institución, sus prácticas no pueden involucrar a una única generación y su marco no puede ser una cultura única. Una nueva escuela, así, debería entender la educación, la construcción de conocimientos, como un proceso interinstitucional, intergeneracional e intercutural (Nilsson y Nocon, 2005).

De acuerdo a estas premisas, una red internacional de universidades, a iniciativa de la Universidad de California, y en la que están implicadas también universidades europeas como la Autónoma de
Barcelona, ha diseñado un modelo en constante evolución a partir de las investigaciones desarrolladas en dicha red. Este modelo, la Quinta Dimensión, tiene como base la participación de estudiantes universitarios realizando prácticas de ApS en entornos comunitarios.

\subsection{Necesidad de transformar las prácticas educativas para favorecer la inclusión}

Partimos de un problema social y educativo concreto. En la década de los 90, la escuela española, claramente monocultural hasta entonces, empieza a experimentar la presencia multicultural en sus aulas. Además de la llegada de un importante contingente de familias inmigrantes procedentes de otros países y otras culturas, se incorporan a las aulas los miembros de una minoría que hasta entonces se había 
mantenido por lo general ajena a a la escuela. Los alumnos gitanos empezaron a acudir a las aulas ordinarias y a llenarlas en los lugares donde sus comunidades estaban asentadas. Hasta entonces llevaban unos 20 años funcionando las escuelas para gitanos en los barrios de barracas y de promociones de vivienda pública (San Román, 1994). Esta nueva estrategia institucional surge como una apuesta por construir una escuela para todos y dejar de lado las escuelas gueto. Sin embargo, los resultados no fueron los esperados. Los índices de fracaso escolar y abandono temprano de esta población siguieron siendo muy superiores a la media (Lalueza y Crespo, 1996; Fernández-Enguita, Mena y Riviere, 2010). Además, muchos de los centros que acogían alumnos gitanos empezaron a ser abandonados por el resto de la población, con lo que el objetivo de integración se venía abajo.

Para intentar explicar este fracaso escolar, que no se da con la misma intensidad en los alumnos de otras comunidades minoritarias, incluso aquellas que tienen lenguas maternas diferentes a la de la escuela (Ferrer, Valiente y Castel, 2008; Vila y Casares, 2009), debemos tener en cuenta las contradicciones inherentes al proceso escolar.

La primera contradicción reside en la falta de sentido de la experiencia escolar en muchos de los niños y niñas de etnia gitana. Esta ausencia de sentido está muy marcada por las discontinuidades, entendidas como diferencias en las metas, valores y prácticas, entre la escuela y las familias, que sostienen distintos 
proyectos evolutivos, y que entran en fuertes contradicciones en nociones como el género, el individuo, el tiempo o la pertenencia (Ogbu, 1987; Greenfield, Raeff y Quiroz, 1998; Lalueza, Crespo, Pallí y Luque, 2001; Carrasco, 2004). Detectar el fundamento de estas discontinuidades supone indagar en los valores culturales no compartidos: mientras que la cultura gitana se fundamenta e $n$ valores decididamente colectivistas, poniendo por delante la comunidad frente al individuo, la escuela es una institución que promueve la individualización y la autonomía personal. Además, esta contradicción se agrava por el desconocimiento hacia el otro, que hace aflorar miedos como la transformación del orden social aceptable desde la propia perspectiva cultural.
La segunda contradicción es histórica y deriva de las relaciones de la comunidad gitana y la cultura mayoritaria, incluyendo las relaciones de poder (Berry, 2001). La escuela, en tanto que institución del grupo dominante, está marcada por esta relación histórica que lleva a la mayoría de las familias gitanas a considerarla como algo ajeno, a veces impuesto, a veces asumido voluntariamente, pero que siempre es un territorio extraño a la comunidad. Así, la relación con la escuela dista mucho de ser una actividad propositiva y más bien está en la base de una actitud de resistencia. Los niños gitanos que se portan adecuadamente en la escuela y tienen un buen rendimiento son acusados de estar apayados (comportarse como no gitanos). Por eso, los escolares gitanos construyen su identidad en 


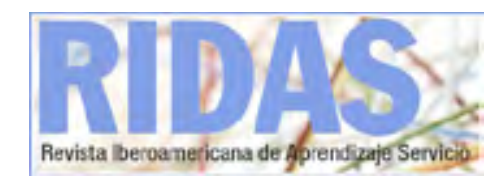

oposición al grupo mayoritario, diferenciándose de sus modos de hacer y de hablar, construyendo diferencias culturales secundarias (Ogbu, 1994).

En estas circunstancias la adaptación de los alumnos gitanos en la escuela es difícil, a la vez que desde la escuela a menudo se asimila la diversidad cultural con déficit, caracterizando a los niños como "carentes de...". Se atribuye, pues, una dificultad personal que debe ser compensada en lugar de entenderse como fruto de las contradicciones entre dos sistemas con prácticas, valores y metas muy distintos, y que ofrecen al niño o la niña gitana proyectos evolutivos divergentes. La interpretación de la diferencia cultural como déficit conlleva una impermeabilidad al contexto, ya que

las dificultades detectadas no promueven que la escuela tenga que transformarse para adaptarse a estos nuevos estudiantes, sino que se mantiene tal y como había funcionando hasta el momento, esperando que sean las familias quienes cambien sus prácticas de crianza, sus valores y sus metas educativas. Con este discurso, hoy predominante en muchas escuelas y en algunas políticas educativas, se busca un sujeto homogeneizado, un niño descontextualizado, que deje su mundo y su forma de vida en la puerta de la escuela porque el lema es "dentro, todos somos iguales". Esta estrategia asimilacionista no ha funcionado, y aún hoy encontramos unos elevadísimos índices de absentismo, abandono temprano y fracaso escolar (Marí-Klose, 2009). 


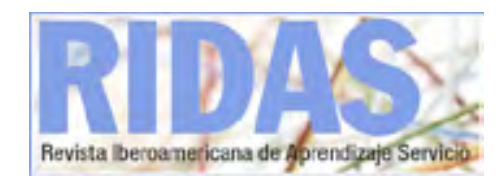

La investigación desde el ámbito universitario, cuando se hace mediante mecanismos de participación con las propias comunidades afectadas, como en la investigación-acción participativa (Montenegro, 2004), tiene la potencialidad de generar sinergias y convertir la investigación en intervención para el cambio. Como veremos más adelante, el Proyecto Shere Rom surge de uno de estos encuentros con la comunidad gitana, y supone la conversión de una investigación en la que ésta comunidad era el sujeto de estudio, en un proyecto compartido con la misma comunidad.

\subsection{Necesidad de afrontar un cambio en la formación universitaria para acercarla a prácticas reales}

Una visita a la vieja Universidad de
Salamanca permite, además de disfrutar de su belleza, captar el origen del modo de entender la práctica universitaria en España. En el centro de las aulas hay un podio en el que un lector dictaba las lecciones a una masa de estudiantes que no gozaban, durante los primeros años de su formación, del privilegio de consultar los libros en la biblioteca. La pesada herencia de un sistema basado en dictar clases sigue pesando demasiado en las concepciones docentes de una parte del profesorado universitario aunque las cosas hayan cambiado mucho.

El acceso al conocimiento supone necesariamente enfrentarse directamente al objeto de conocimiento. Además de saber cómo se trabaja en la práctica profesional derivada de la formación 
universitaria, es necesario involucrarse en esa práctica. En esta línea, las prácticas externas o metodologías docentes como el aprendizaje basado en problemas $(A B P)$ contribuyen a dotar de mayor significado a la práctica universitaria. Las primeras sitúan al estudiante en el rol de aprendiz en el sentido clásico que recupera Rogoff (2003) para explicar los procesos de aprendizaje mediante la participación guiada. Un modelo universal que precede en el tiempo a la escuela y que sigue siendo el modo privilegiado de incorporación a las prácticas profesionales y laborales. Por otro lado, el ABP permite que el estudiante, en lugar de intentar reproducir el discurso dado por el profesor o dar con la respuesta correcta a una pregunta del mismo, indague sobre diferentes opciones para resolver un problema, buscando activamente las fuentes de información, no para reproducirlas, sino para obtener de ellas los recursos necesarios para resolver el problema (Araújo y Sastre, 2008).

Pues bien, el ApS en la Universidad, entendido como un conglomerado de prácticas educacionales donde convergen el trabajo comunitario y la educación formal, permite conjugar algunas de las propiedades de las prácticas externas y del ABP. Así, al igual que las prácticas externas, genera un aprendizaje situado ya que se lleva a cabo en un contexto real. De este modo, está presente el anclaje contextual que da sentido a todo conocimiento (LCHC, 1982), lo que no se garantiza a través de una formación exclusivamente académica. Operar con problemas reales supone 
en sí una práctica profesionalizadora en tanto que desarrolla competencias relacionadas con la acción. Pero además, el ApS incorpora un importante elemento del ABP: esta acción está orientada a una meta, está motivada más allá del propio proceso de aprendizaje. La meta que se le presenta al estudiante que participa en un proyecto de ApS no es en primer lugar aprender, sino resolver un problema real, producir una mejora social. Ello supone también aprendizaje, pero no se orienta a sí mismo, sino al exterior. Los conocimientos y las competencias se adquieren de manera no desligada de la realidad social que les da sentido.

Estas dos características, aprendizaje situado y aprendizaje orientado a metas, son los elementos principales delaprendizaje auténtico, que se produce siempre en escenarios de actividad específicos y adquiere significado personal cuando es asimilado dentro de la propia historia de vida del aprendiz. El aprendizaje auténtico tiene lugar cuando el aprendiz se encuentra inmerso en una comunidad de práctica (Wenger, $2001)$ y debe posicionarse e implicarse personalmente en el proceso mismo del aprendizaje. Se produce entonces un cambio identitario en el que su posición en el mundo se transforma (Wortham, 2006). El aprendizaje auténtico activa competencias, tradicionalmente poco desarrolladas en ámbitos universitarios, tales como el compromiso social y la iniciativa personal en la aplicación de los conocimientos académicos a problemas reales. En este proceso de 
aprendizaje, la reflexión sobre la participación y el propio proceso de aprendizaje constituyen una dimensión fundamental de su metodología (Giles y Eyler, 1994). Un aprendizaje realizado en comunidad hace posible esta asimilación personal, junto con la adquisición de habilidades cognitivas, de manera que el aprendizaje auténtico implica la transformación de la persona global, y se relaciona con la adquisición de una nueva forma de mirar al mundo, una nueva forma de verse a sí mismo.

\section{Descripción de la experiencia: 16 años del Proyecto Shere Rom}

El Proyecto Shere Rom es un proyecto interinstitucional, intergeneracional e intercultural, que supone la participación de diversos colectivos:

a) Estudiantes de Ia UAB.
Participan entre 110 y 140 estudiantes cada curso, la mayoría a lo largo de un semestre y una minoría durante todo el curso.

b) Niños, niñas y adolescentes, entre 6 y 14 años, en sus escuelas e institutos durante horario escolar o en centros comunitarios en horario extraescolar. Participan cada año entre 250 y 300. Muchos de ellos de manera continuada durante tres o cuatro años.

c) Maestros, educadores y agentes comunitarios.

d) Profesores de la Facultad de Psicología e investigadores del grupo de investigación DEHISI.

El proyecto se inició como una experiencia local en 1998, en el barrio 


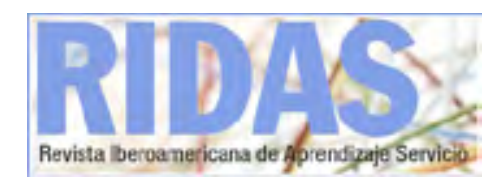

de Sant Roc de la ciudad de Badalona, del área metropolitana de Barcelona. En este barrio, vivían algo más de doce mil personas, de las que cerca de la mitad eran de etnia gitana. Como dato relevante sobre el nivel de estudios formales nos encontramos, de acuerdo al censo, que el $9,4 \%$ de la población era analfabeta $y$, más significativo aún, entre los jóvenes que en 1998 tenían entre 18 y 22 años había un 0,9\% de analfabetismo, a los que hemos de añadir un $9,1 \%$ que no llegaban a completar ni siquiera los 6 cursos de primaria. Si unimos a estos dos grupos los que no habían conseguido el graduado escolar tenemos el $48 \%$ de los jóvenes de ese grupo de edad (Lalueza, Crespo, Barrientos y Luque, 2001).

A partir de un proyecto de

investigación anterior, sobre las prácticas educativas familiares en la comunidad gitana, se había iniciado un proceso de colaboración entre un equipo de investigación de la Universitat Autònoma de Barcelona con la Asociación Gitana de Badalona (Crespo, Lalueza y Pallí, 2002). El Proyecto Shere Rom se construye a partir de un largo proceso de conversaciones y negociaciones entre ambos colectivos, del que emergieron metas compartidas. Estas conversaciones entre miembros de la asociación y de la universidad se convirtieron en un diálogo intercultural para la definición de una actividad educativa que rompiera con dos contradicciones: Ias discontinuidades familia-escuela (Bereményi, 2011; Greenfield, Raeff y Quiroz, 1998; Lalueza, 2012) y las relaciones históricas entre 


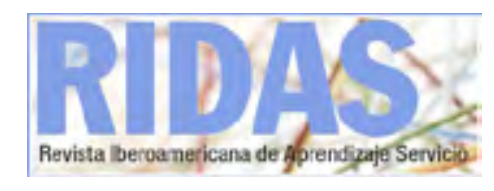

comunidades (San Román, 1994), al mismo tiempo que se alejaba de la teoría del déficit y los objetivos asimilacionistas para construir una comunidad de práctica fuera de la escuela, dónde colaboraran participantes de los distintos grupos culturales, dónde todos fueran considerados interlocutores válidos y existiera una flexibilidad en los roles.

Para que la actividad cumpliera con la función educativa que se esperaba de ella, ésta debía ser significativa para todos los participantes, los objetivos debían ser discutidos entre todos y las metas debían ser claras. La actividad debía estar orientada a proporcionar a las niñas y niños de la comunidad herramientas que les permitieran superar el fracaso escolar generalizado. Pero también debía transformar la percepción de la

escuela como una institución lejana, ajena y poco permeable a las familias gitanas. Esta transformación sólo era posible a partir de una iniciativa de la propia comunidad.

Finalmente, se planteaba también la necesidad de modificar la concepción de los gitanos como sujetos pasivos del proceso de escolarización, y situarlos como agentes de un proceso de cambio educativo que incluyera la diversidad cultural en la escuela. Este objetivo común se materializó en la creación de la Casa de Shere Rom, una comunidad de práctica gestionada por la Asociación Gitana, ubicada en sus propios locales, y diseñada y asistida por el equipo de investigación de la UAB.

Este proyecto se ha implementado, con un formato muy similar, en otras dos asociaciones gitanas de la ciudad 


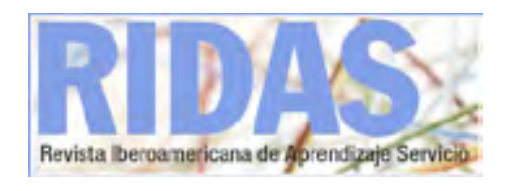

de Barcelona (en los barrios de Gràcia y Hostafrancs), en un centro cívico (barrio de Roquetes) y en la biblioteca pública del barrio de Bon Pastor. En cada uno de estos entornos se ha seguido un proceso de negociación con la comunidad para construir proyectos ajustados al contexto, a las necesidades detectadas y con el fin de crear objetivos compartidos.

\section{La Quinta Dimensión, un modelo de actividad para el proyecto de Shere Rom}

La Casa de Shere Rom se inspiró en una red de comunidades de prácticas desarrolladas por Michael Cole y el Laboratory of Comparative Human Cognition de la Universidad de California, y más concretamente en una de estas comunidades, La Clase Mágica ( Vásquez, 2002 ). La articulación de esta última como espacio propio de una comunidad cultural (latina en este caso), nos ofreció un modelo ideal para el diseño del proyecto que pretendíamos llevar a cabo en los locales de la Asociación Gitana de Badalona.

La Quinta Dimensión es un modelo de actividad educativa basado en el aprendizaje colaborativo mediado por las TIC, y basado en la colaboración intergeneracional e interinstitucional (Cole, 1999; Nilsson y Nocon, 2005). Pretende construir un contexto de actividad donde la utilización creativa de las TIC permita desarrollar las competencias necesarias para la inclusión escolar de los participantes, y donde se pongan al alcance de la población joven nuevas formas de utilización de las TIC. Su desarrollo se da dentro y fuera de la escuela, pero en todos los casos se orienta a la creación de un entorno donde los 
participantes comparten significados, metas y herramientas.

Los proyectos inspirados en este modelo se fundamentan en el marco teórico de la Psicología Cultural, así como en aportaciones de la Psicología Social Crítica y la práctica de la Psicología Comunitaria. Sus principios se pueden resumir en los siguientes puntos:

1. La actividad educativa debe estar vinculada a la comunidad donde se lleva a cabo, con el fin de que el aprendizaje sea significativo y esté conectado a los objetivos comunitarios. Los niños y sus familias deben de ser respetados y considerados como interlocutores con capacidad para formular y perseguir unos objetivos $\mathrm{y}$, por lo tanto, para transformar la actividad.

2. La construcción del conocimiento es un proceso social basado en la participación y la colaboración entre actores que poseen conocimientos y experiencias distintas. Su encuentro, bajo determinadas condiciones, se produce en la Zona de Desarrollo Próximo, en la cual el aprendiz, mediante la guía del experto, adquiere progresivamente la autonomía en nuevas tareas.

3. Los roles de experto y aprendiz son flexibles, y un mismo sujeto puede transitar entre uno y otro según los objetivos y las actividades concretas.

Las actividades del Proyecto Shere 
$R \circ m$ se desarrollan en espacios equipados con ordenadores y periféricos conectados a Internet, en los que chicas y chicos de la comunidad colaboran con estudiantes universitarios y educadores en la resolución de tareas, normalmente (pero no exclusivamente) ante la pantalla de un ordenador.

La lectura y la escritura son herramientas fundamentales que se utilizan siempre con una meta explícita. Se lee para informarse de algo relacionado con la actividad, se escribe siempre para una audiencia, sea un compañero, un personaje virtual, los potenciales lectores de un blog o los espectadores de sus creaciones.

Cuando un niño empieza por primera vez la actividad, un estudiante le ayuda a hacer una carta para un personaje misterioso, que nadie ha visto pero que responde las cartas, participa en chats y envía mensajes a foros compartidos. Este personaje es una ambigua y misteriosa figura que se relaciona y se comunica de diferentes maneras con los participantes en la comunidad de prácticas. La identidad del Mago es desconocida y se utiliza para estimular la imaginación y el razonamiento de los chicos. Además, a menudo se usa para mediar en conflictos y conducir la atención hacia otros contextos.

En función de las competencias logradas por los miembros del grupo, la actividad se desarrolla según dos modelos: Laberinto y Trovadores.

E I Laberinto es una actividad concebida para introducir a los participantes en el uso de las TIC a 
través de la colaboración con compañeros expertos. Éstos son los estudiantes universitarios que trabajan con uno o dos niños en la resolución de tareas. Para participar se parte de la representación gráfica d e un laberinto que conecta varias habitaciones, y en cada una de ellas hay una 0 varias propuestas de actividad que los niños y sus acompañantes universitarios tendrán que llevar a cabo. Cada actividad tiene una guía de tareas con tres niveles de complejidad que explica qué se tiene que hacer una vez iniciado el juego. Cuando se llegan a cumplir los requisitos de uno de los dos últimos niveles, se puede avanzar a la siguiente casilla del laberinto e iniciar un nuevo juego. El viaje que el niño hace a lo largo de los días a través del laberinto, se registra en un pasaporte personal. El estudiante universitario es el compañero que puede ayudar a resolver la tarea, dar pistas, facilitar apoyo, proporcionar el lenguaje adecuado... Al final de cada día de actividad, éste escribe un diario de campo donde se detallan los progresos y las dificultades encontradas, siendo estos diarios una herramienta fundamental para la reflexión sobre su propio proceso de aprendizaje y sobre la puesta en práctica de las competencias adquiridas en las aulas universitarias.

Trovadores es un modelo orientado a la creación de pequeños relatos digitales para dar a las niñas y niños la oportunidad de ser creadores y participantes activos en su proceso de aprendizaje. Se organiza en grupos de tres o cuatro niños acompañados por un estudiante universitario y entre todos ellos elaboran 
presentaciones dinámicas, animaciones o películas de vídeo de corta duración sobre un tema escogido por el propio grupo. El proceso de elaboración del relato se realiza de forma colaborativa y con la ayuda de herramientas como cámaras y programas de edición. El proceso de realización pasa por diferentes fases: planificación del trabajo a realizar, selección de la temática, elaboración del guion, grabaciones, edición y finalmente visualización. Pero este proceso no es lineal sino en espiral. Es decir, cada visualización lleva a la revisión de lo que se ha hecho, de forma que no hay una evaluación externa, sino una reflexión permanente de los autores sobre su obra. Libertad para escoger, actividad colaborativa y trabajo orientado a una audiencia son los tres ejes fundamentales de esta actividad.
Cada año concluye con una visita de los chicos y chicas mayores de cada centro al campus universitario para presentar los productos audiovisuales desarrollados en los proyectos de trovadores. Los estudiantes universitarios colaboran en la preparación y actúan como anfitriones, guiando a los chicos en pequeños grupos por las instalaciones de la facultad. El curso finaliza así, con este encuentro en la Universidad, y con una comida al aire libre que reúne niños, estudiantes universitarios, maestros, agentes comunitarios e investigadores de la Universidad.

La Casa de Shere Rom ha sido y es un laboratorio para el diseño de nuevas prácticas educativas y estrategias de empoderamiento de la comunidad gitana, y ha mostrado su 
potencial para mejorar las competencias de los niños y niñas que asistían a ella (Padrós, SánchezBusqués, Lalueza y Crespo, 2014). Después de cinco años, ya disponíamos de un modelo que parecía dar buenos resultados, pero los niños asistían únicamente dos horas a la semana mientras que pasaban en la escuela jornadas completas y las competencias que habían adquirido en la Casa de Shere Rom no parecían transferirse automáticamente a ésta. Así, necesitábamos incidir en la misma escuela para poder transformar sus prácticas. Para ello disponíamos del modelo que habíamos ido desarrollando a lo largo de esos cinco años en un entorno libre de las trabas propias de las instituciones de educación formal. En el año 2004 empieza de este modo el proyecto
Shere Rom en la Escuela.

La entrada en la escuela implica incorporarnos a una institución con unas reglas, unos roles, unas prácticas y unos objetivos muy distintos a los de las comunidades gitanas. Así que tuvimos que buscar maneras de mantener los objetivos que habíamos detectado como básicos, pero adaptándonos al nuevo contexto y a sus dinámicas. Este nuevo contexto institucional nos permitía acercarnos a los maestros, a sus prácticas y sus discursos (Lamas y Lalueza, 2012), e intentar plantear transformaciones que rompieran las discontinuidades antes planteadas (Essomba, 2003). Ahora los interlocutores, con los que se discutía sobre las actividades y sobre qué objetivos teníamos, eran maestros, y por lo tanto, nos encontrábamos ante 
nuevos retos.

E I proyecto Shere Rom se ha introducido en varias escuelas e institutos que tienen una parte importante de población de etnia gitana (aproximadamente la mitad de los alumnos); inmigrantes de distintos países, sobretodo Pakistán, India, Marruecos, China, Ecuador y Perú; y un número marginal de autóctonos del grupo mayoritario. Así pues, los niños ya no eran solamente de cultura gitana, sino que una mayor diversidad cultural estaba presente en el aula y el proyecto que nosotros ofrecíamos debía contemplarla.

Esta experiencia ha permitido seguir una serie de transformaciones tanto en los niños como en la dinámica del centro, fruto de la introducción de nuevas formas de enseñanza y aprendizaje basadas en la colaboración, nuevos roles con la introducción de estudiantes universitarios y nuevas formas de organización del aula y coordinación para la acción.

Pero no se ha tratado simplemente de trasladar o implantar el modelo Quinta Dimensión en la escuela. El resultante es un tipo de actividad diferente, nueva, un tercer objeto en términos de Engeström (2007), fruto de la contraposición del modelo 5D con el modelo clásico escolar. El mayor logro ha consistido en transformar la práctica de los maestros implicados, que han transitado desde un modelo tradicional de enseñanza basado en la transmisión a formas diversas basadas en la participación y la colaboración, proceso que no se ha dado sin dificultades y contradicciones 


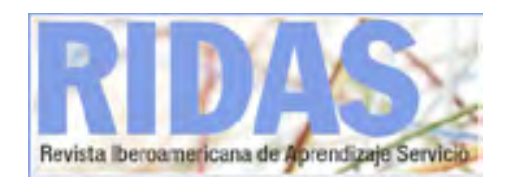

(ver un estudio en detalle en Lamas y Lalueza, 2012).

Durante los últimos años el proyecto Shere Rom se ha configurado como una red de actividades escolares y extraescolares coordinadas desde la Universitat Autònoma de Barcelona, de manera similar al modelo UClinks de la Universidad de California. En estos momentos hay en funcionamiento ocho espacios del Proyecto Shere Rom, en tres escuelas de enseñanza primaria, un instituto de secundaria, dos locales gestionados por comunidades gitanas y dos centros cívicos de barrio. Funcionan autónomamente y se desarrollan de forma que presentan grandes diferencias entre si, siempre buscando la adaptación en los respectivos contextos, pero se articulan como una red que se puede visualizar en la comunidad virtual 5D Barcelona

(http://www.5dbarcelona.org).

\section{Modelo de participación de los estudiantes universitarios en ApS}

E I Proyecto Shere Rom intenta dar una respuesta a las necesidades planteadas al principio de este artículo. En primer lugar, como proyecto de investigación, contribuye, dentro de la red internacional Quinta Dimensión, en la construcción de modelos educativos alternativos al viejo modelo de transmisión, basados en la colaboración, el uso de las TIC, y la presentación ante los alumnos de las tareas de aprendizaje como propuestas de indagación y de creación. En segundo lugar, como proyecto de intervención ofrece un modelo de actividad inclusivo, en el que los miembros de comunidades 
minoritarias (en este caso básicamente los gitanos), no son usuarios pasivos, sino participantes que contribuyen en la construcción del modelo. $Y$ en tercer lugar, en tanto que proyecto docente, introduce a los estudiantes universitarios en una práctica real en la que a partir de su intervención aprenden sobre los procesos de desarrollo, aprendizaje y participación social, al tiempo que su actividad tiene un impacto social. Aquí vamos a desarrollar esta última vertiente, la de la participación de los estudiantes en el proyecto como práctica de aprendizaje servicio.

Los proyectos de ApS de la Facultad de Psicología de la UAB se gestionan desde la entidad Servicios y Proyectos Estratégicos de Psicología (SIPEP), cuya función es desarrollar vínculos entre la facultad y la sociedad, mediante el establecimiento de acuerdos y convenios que permitan la prestación de servicios a la comunidad. El Proyecto Shere Rom es el primero de ApS, coordinado por un grupo de investigación de la Facultad y con la cobertura de un convenio con el Ayuntamiento de Barcelona, por mediación del Consejo de Participación del Pueblo Gitano de Barcelona. En años anteriores ha obtenido premios y financiación de la Fundació Jaume Bofill y de la Fundació Societat Econòmica Barcelona d'Amics del País. Para la implementación del proyecto se han generado nuevas herramientas de trabajo que se han desarrollado gracias a los proyectos de investigación financiados por la Unión Europea, el Ministerio de Educación y la Generalitat de Catalunya. 
Cada semestre participan entre 50 y 70 estudiantes universitarios para completar los créditos de prácticas de algunas asignaturas, del Grado de Psicología, vinculadas con el desarrollo humano (Psicología Evolutiva y Psicología Cultural) o con la intervención social (Intervención Psicosocial y Comunitaria e Infancia y Familias en Contextos de Dificultad). Todos ellos tienen la opción de hacer unas prácticas de aula menos exigentes en tiempo y compromiso, de modo que su participación, aunque reconocida académicamente, tiene un cierto grado de voluntariedad.

Cuando inician su participación en el proyecto ingresan en una comunidad virtual donde encuentran todas las herramientas y guías de actividad de cada centro, que les servirán como medio de comunicación entre los integrantes de su grupo y como herramienta a compartir con los niños.

La formación específica que reciben para el desarrollo de sus tareas se da en tres ámbitos. El primero consiste en tres sesiones de seminario: la primera, previa al inicio de la actividad, dedicada a introducir el modelo educativo de la Quinta Dimensión; la segunda, tras un rodaje en la actividad de tres semanas, se orienta a analizar las formas de interacción en un contexto de aprendizaje colaborativo; y la tercera, en el ecuador de las actividades, sobre las especificidades culturales de los participantes y las relaciones con la escuela.

El segundo ámbito consiste en el feedback que reciben después de cada jornada de actividad por parte 
del educador o investigador a cargo de la coordinación de la misma. Allí se analizan las dificultades de la interacción con los niños, las incidencias en el desarrollo de las tareas y los aprendizajes realizados sobre la práctica. Por último, el tercer ámbito supone para los estudiantes rellenar unos diarios de campo que reciben la retroalimentación del profesorado y las sesiones de tutoría con el profesor de referencia de la materia que cursan.

Hay un segundo nivel de participación, ligado al prácticum de la titulación, de estudiantes más expertos, tan sólo tres o cuatro que durante un año participan en la gestión del proyecto (coordinación, evaluación y rediseño de actividades). $Y$ un tercer nivel, de estudiantes de postgrado, en número similar, que participan en las tareas de investigación.

\section{Lo que los estudiantes aportan al proyecto}

El rol de los estudiantes en el proyecto es fundamental, ya que sin ellos no podría llevarse a cabo, no sólo por su aportación como recursos humanos, sino porque precisamente las características de un grupo como es el de los estudiantes universitarios permite incorporar un rol singular e imprescindible en el proyecto: la figura de un experto que juega un rol de compañero.

Así, en primer lugar, el estudiante posibilita una experiencia de aprendizaje colaborativo. Existe un claro desequilibrio en conocimientos entre el niño y el estudiante, pero el niño juega en su terreno, es un miembro antiguo de la institución 
frente al estudiante recién llegado, de manera que hay una cierta flexibilidad en los roles de experto y aprendiz, lo que resulta ideal en la construcción de la zona de desarrollo próximo (Vygotsky, 1978).

En segundo lugar, el estudiante es también uno de los artefactos que median en el aprendizaje del niño, es decir, se sitúan entre el sujeto y el objeto en el proceso de aprendizaje como ocurre con las diversas herramientas TIC. Como artefacto mediador, transforma el objeto de conocimiento y también al sujeto. Por lo tanto, genera una situación nueva, inédita en el aula. Pasamos de la situación clásica de un maestro y un grupo de alumnos, a la de un maestro (que puede no estar presente), herramientas TIC, artefactos específicos como el mago y las guias de tarea y estudiantes que cooperan con parejas o grupos de niños. En esta cooperación el estudiante proporciona el andamiaje necesario para que el niño realice la tarea corrigiendo errores y disponiendo de pistas que le faciliten sus logros. Pero también se constituye en un narrador de la actividad, en alguien que relata los acontecimientos ligados a las tareas de manera que adquieran un sentido. De este modo el estudiante contribuye a dar significado a la actividad, a definir las metas de la misma, a organizar las acciones necesarias, en suma, a regular la conducta del niño.

En tercer lugar, el estudiante es un modelo lingüístico en tanto que dispone del lenguaje adecuado para entornos académicos, apto para comprender las tareas a realizar. Pero 
el tipo de relación personalizada, y el carácter poco institucional de la participación del estudiante permite un uso flexible del lenguaje, de manera que se generan episodios de traducción entre el lenguaje social de los niños y el lenguaje social del mundo académico -en el sentido que da Bakhtin al término lenguaje social-, algo imprescindible en la educación intercultural.

En cuarto lugar, la presencia del estudiante permite la creación de un vínculo afectivo importante en todo proceso de aprendizaje. La curiosidad que los niños sienten por estos estudiantes, menos distanciados en edad que otros referentes adultos, que se presentan como un compañero para llevar adelante una serie de tareas, se transforma habitualmente en un vínculo afectivo que facilita la actividad y que contribuye a dotarla de sentido.

En quinto lugar, en tanto que se ha establecido un vínculo, el estudiante funciona potencialmente como modelo vital. En entornos en los que escasean modelos de trayectoria académica de éxito, la cercanía del estudiante abre nuevas perspectivas en la construcción de la identidad del niño. En este sentido, el estudiante contribuye a mostrar otros mundos posibles al niño, le da cuenta de otro tipo de experiencias que aquellas a las que accede a través de la familia y la escuela. En otras palabras, le informa de un mundo más amplio.

En sexto lugar, más allá de su impacto en los niños con los que toma contacto, el estudiante contribuye al cambio cuando opera en el aula de una escuela. El rol jugado 
por los estudiantes en un primer momento diluye el rol clásico del maestro, pero poco a poco facilita que éste vaya construyendo un nuevo rol, no ya el de transmisor, sino nuevas facetas del rol de maestro, como la planificación, la tutorización, la dinamización grupal, la observación, etc.

Por último, en séptimo lugar, el estudiante contribuye a recopilar los datos necesarios para la investigación y el proceso de innovación a través de las notas recogidas en sus diarios de campo.

\section{Lo que el proyecto aporta a los estudiantes}

Los diarios de campo y las memorias entregadas por los estudiantes durante los 16 años de funcionamiento del proyecto nos han permitido recoger elementos de autoevaluación que nos permiten esbozar un cuadro de competencias adquiridas, siempre desde la perspectiva de los propios estudiantes.

En primer lugar, como el elemento más presente en las memorias de los estudiantes, aparece una dimensión ética y social. Toma de conciencia de la diversidad social y cultural, revisión de prejuicios, descubrimiento de la alteridad, necesidad de la intervención para el cambio social, son algunas de las adquisiciones a las que se refieren.

En segundo lugar, aparecen referencias a la adquisición de competencias en una dimensión personal y actitudinal. Mejora de la confianza en las propias capacidades de relación, de superación de conflictos, de relación. En los relatos 
de los estudiantes se presenta a menudo como un impacto en la identidad personal.

En tercer lugar, también presente en la mayoría de las memorias, aparece una dimensión profesional que da cuenta de competencias relacionadas con la práctica de la titulación que están cursando: comunicación con niños, comunicación intercultural, dinamización de sesiones, conducción de grupos y aplicación de conocimientos académicos a la realidad.

Por último, en menor medida probablemente por el diseño de este tipo de práctica, aparece una dimensión en emprendimiento, con competencias relacionadas con la gestión: dinamización social, planteamiento de alternativas, diseño de planes de acción o gestión de proyectos.

Al menos las tres primeras dimensiones nos muestran las potencialidades de aprendizaje mediante la participación en este proyecto, un aprendizaje que supone cambios en el sujeto: aprender sobre la realidad del entorno y adquirir compromisos; aprender sobre uno mismo y sobre sus potencialidades; y aprender sobre la práctica profesional en el trabajo con personas y comunidades.

Queda por desarrollar más a fondo la dimensión relacionada con el emprendimiento social. Consideramos que el diseño de la actividad, ya prefigurado en las negociaciones entre el equipo de la universidad, las escuelas y las entidades comunitarias, no deja mucho espacio para el estudiante de grado que 
centra su actividad en la relación con niños y adolescentes. Sin embargo, los estudiantes que han participado en un segundo y tercer nivel (coordinación e investigación) han mostrado evidencias de adquisición de competencias de este tipo, como consecuencia de su participación en el diseño y la gestión del proyecto.

Hasta el momento, el proyecto Shere Rom ha demostrado ser sostenible durante un largo período de tiempo, y haber contribuido en los ámbitos de la investigación, la docencia y el retorno social gracias a un diseño en el que el ApS juega un papel central. El siguiente reto consiste en incrementar el grado de participación de los estudiantes en las tareas de diseño de tareas y planificación, en la línea de crear una experiencia ApS más auténtica.

\section{Referencias bibliográficas}

Araújo, U y Sastre, G. (Coords.) (2008). El aprendizaje basado en problemas. Una nueva perspectiva de la enseñanza en la universidad. Barcelona: Gedisa.

Bereményi, B. (2011). Intercultural policies and teachers' contradictory views. The Roma case en Catalonian schools. Intercultural Education Journal, 22(5), 355-369.

Berry, J. (2001). A Psychology of Immigration. Journal of Social Issues, 57(3), 615-631.

Carrasco, S. (2004). Inmigración, contexto familiar y educación. ICE UAB: Cerdanyola del Vallès.

Cole, M. (1999) Cultural psychology: Some general principles and a concrete example. En Yrjö Engeström, 
Reijo Miettinen y Raija - Leena Punamäki (Eds.), Perspectives on activity theory. Cambridge, UK: Cambridge University Press.

Crespo, I.; Lalueza, J.L. y Pallí, C. ( 2002 ). Moving communities: a process of negotiation with a gypsy minority for empowerment. Community, Work and Family, 5(1), 49-66.

Crook, C. (1991) Computers in the zone of proximal development: Implications for evaluation. Computers Education, 17(1), 81-91.

Engeström, Y. (1999) Activity theory and individual and social transformation. Yrjö Engeström, Reijo Miettinen y Raija - Leena Punamäki (Eds.), Perspectives on activity theory. Cambridge, UK: Cambridge University Press.
Engeström, Y. (2007). Enriching the Theory of Expansive Learning: Lessons From Journeys Toward Coconfiguration. Mind, Culture and Activity, 14 (2), 23-39.

Essomba, M A. (2003). Educación e inclusión social de inmigrados $y$ minorías. Praxis: Barcelona.

Fernández - Enguita, M; Mena, L.; Riviere, J. (2010). Fracaso y abandono escolar en España. Colección estudios sociales, 29. Barcelona: Obra social Fundación "la Caixa".

Ferrer, F; Valiente, Ò; y Castel, J L. ( 2008 ). Equitat, Excel.lència $i$ eficiència educativa a Catalunya. Una anàlisis comparada. Barcelona: Fundació Bofill.

Gatto, J. (2000). A different kind of 
teacher: Solving the crisis of American schooling. Berkeley, CA: Berkeley Hills Books.

Giles, D.; Eyler, J. (1994). The theoretical roots of service-learning in John Dewey: Toward a theory of service-learning. Michigan Journal of Community Service Learning, 1 (1), 77-85.

Greenfield,P; Raeff, C; Quiroz, B. (1998). Cross - Cultural conflict in the social construction of the child. Aztlan, The Journal of Chicano Studies, 23, 115-125.

Laboratory of Comparative Human Cognition (LCHC). (1982). A model system for the study of learning difficulties. Quarterly Newsletter of the Laboratory of Comparative Human Cognition, 4, 39-66.
Lalueza, J.L. .(2012). Modelos psicológicos para la explicación de la diversidad cultural. Cultura y Educación 24(2), 149 -162.

Lalueza, J.L.; Crespo, I. (1996). Some difficulties arisen by the cultural bias in the study of human development. Cultura y educación, 3, 51-69.

Lalueza, J.L.; Crespo, I; Barrientos, A; Luque, M.J. (2001). Estudio de necesidades sociales del barrio de Sant Roc. Estudio encargado por la Generalitat de Catalunya.

Lalueza, J.L.; Crespo, I; Pallí, C. y Luque, M.J. (2001). Socialization and cultural change in a minority ethnic community. The gypsy developmental niche. Cultura y educación, 13(1), 115-130. 
Lalueza, J.L.; Lamas, M. (2012). Apropiación de un modelo colaborativo en escuelas multiculturales con alumnado en situación de exclusión social. Cultura y Educación, 24(2), 177-191.

Marí-Klose, P (Coord.). (2009). Informe de la Inclusión Social en España 2009. Barcelona: Fundació Caixa de Catalunya.

Moll, L; Amanti, C; Neff, D; Gonzalez, N. (1991). Funds of knowledge for teaching: Using a qualitative approach to connect homes and classrooms. Theory Into Practice, 31(2), $132-141$.

Montenegro, M. (2004). La investigación acción participativa. En Gonzalo Musitu, Juan Herrero, Leonor M. Cantera y Marisela Montenegro: Introducción a la psicología comunitaria. Barcelona: Editorial UOC.

Nilsson, M; Nocon, H. (2005). School of Tomorrow. Teaching and Technology in Local and Global Communities. Berna: Peter Lang.

Ogbu, J. (1987). Variability in Minority School Performance. A Probleme in Search of an Explanation. Anthropology and Educacion Quarterly, 18, 312-334.

Ogbu, J. (1994). From Cultural Differences to Differences in Cultural frame of Reference. In Patricia M. Greenfield and Rodney R. Cocking (Eds.): Cross-cultural Roots of Minority Child Development (365391). Hillsdale: LEA.

Padrós, M.; Sànchez-Busqués, S.; Lalueza, J.L. y Crespo, I. (2014). The 


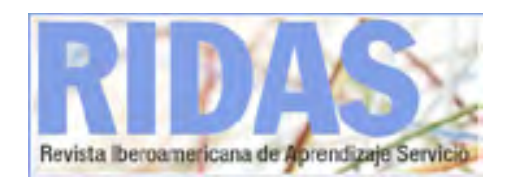

Shere Rom Project: Looking for Alternatives to the Educational Exclusion of Roma. International Journal for Research on Extended Education Vol 2014 (2), 46-62

Reyes, I.; Esteban-Guitart, M. (2013). Exploring Multiple Literacies from Homes and Communities. A Crosscultural Comparative Analysis. International Handbook of Research on Children's Literacy, En Kathy Hall, Teresa Cremin, Barbara Comber y Luis Moll. Learning and Culture. John Wiley y Sons.

Rogoff, B. (1994). Developing understanding of the idea of communities of learners. Mind, culture, and Activity, 1(4). 20-29.

Rogoff, B. (2003). The Cultural Nature of Human Development. Oxford NY: Oxford University Press.
Säljö, R. (2000). Lärande i praktiken: Ett sociokulturellt perspektiv (Learning in practice: A sociocultural perspective). Stockholm: Prisma.

San Román, T. (1994). La diferència inquietant. Barcelona: Alta Fulla.

Vásquez, O. (2002). La Clase Mágica: Imagining Optimal Possibilities in a Bilingual Community of Learners. New Jersey: Laurence Erlbaum Publishers.

Vila, I; Casares, R. (2009). Educación y sociedad. Una perspectiva sobre las relaciones entre la escuela y el entorno social. Barcelona: Horsori Editorial, S.L.

Vygotsky, L. (1978). Mind in society. Cambridge, MA: Harvard University Press.

Wenger, E. (2001). Comunidades de práctica. Aprendizaje, significado e 


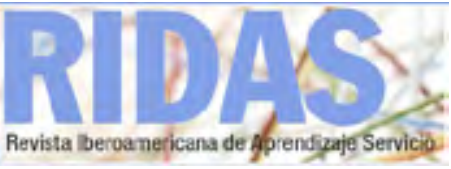

identidad. Barcelona: Paidós.

identification and academic learning.

Wortham, S. ( 2006$)$. Learning New York: Cambridge University identity: the joint emergence of social Press.

Lalueza, J.L.; Sánchez-Busqués; S. y Padrós, M. (2016). Creando vínculos entre universidad y comunidad: el proyecto Shere Rom, una experiencia de aprendizaje servicio en la Facultad de Psicología de la Universitat Autònoma de Barcelona. RIDAS, Revista Iberoamericana de Aprendizaje Servicio, 2, 3369. DOI10.1344/RIDAS2016.2.3 